(C) 2017 IEEE. Personal use of this material is permitted. Permission from IEEE must be obtained for all other uses, in any current or future media, including reprinting/republishing this material for advertising or promotional purposes, creating new collective works, for resale or redistribution to servers or lists, or reuse of any copyrighted component of this work in other works. 


\section{The Detection and Suppression of Unbalanced Magnetic Pull in Wound Rotor Induction Motors Using Pole-Specific Search Coils and Auxiliary Windings}

\author{
David Dorrell \\ Senior Member, IEEE \\ University of KwaZulu Natal \\ Durban 4041, South Africa \\ Dorrelld@ukzn.ac.za
}

\author{
Ahmad Salah \\ Member, IEEE \\ University of Technology Sydney \\ Sydney, NSW 2007, Australia \\ Ahmad.a.salah@student.uts.edu.au
}

\author{
Youguang Guo \\ Senior Member, IEEE \\ University of Technology Sydney \\ Sydney, NSW 2007, Australia \\ Youguang.Guo-1@uts.edu.au
}

\begin{abstract}
This paper puts forward a concept for measuring rotor eccentricity in a wound rotor induction machine. The analysis is fully developed in the theory section then an experimental rig is described and measurements are taken. These are compared to the calculated values. Pole-specific search windings are necessary in a wound rotor machine because standard sideband current measurement techniques are only valid for cage induction machines. The search coils can also be used to suppress unbalanced magnetic pull.
\end{abstract}

Index Terms-- Condition monitoring, DFIG, eccentric rotors, unbalanced magnetic pull, wound rotor induction machines

\section{INTRODUCTION}

When the rotor in an electrical machine is not centered in the stator bore it is generally called rotor eccentricity and can cause radial forces, vibration and bearing wear. It has recently been extensively studied in permanent magnet machines [1], [2]. Condition monitoring methods are also used to monitor developing faults, such as rotor eccentricity [3]-[5]. The normal method is to assume that the rotor is offcenter by a constant amount down from the axial length of the machine, although [6] investigated a more realistic scenario where the eccentricity varies down from the axial length of the machine. When the rotor is not centered in an induction motor the unbalanced magnetic pull (UMP) can vary substantially with voltage and load [6] and this can be difficult to assess. In this paper a wound rotor machine is investigated; the UMP in this type of machine can vary greatly from its cage-rotor equivalent [7] and it is substantially higher. Fig. 1 illustrates this with a breakdown of the airgap magneto-motive force (MMF) and flux waves in terms of a 4 pole machine and this figure shows the interaction of these. The main difference is the lack of 2 pole and 6 pole MMF waves on the rotor that can damp the additional flux waves generated by the rotor eccentricity, hence reducing the UMP. This is because the cage structure has many effective parallel paths which evens out the airgap flux and reduces the radial pull. The use of additional damper windings has been investigated [7]-[10] and a similar theme is investigated here. However, this is combined with using pole specific search coils to assess the degree of eccentricity.

Rotor eccentricity has been defined as either static (rotor offset from the stator bore but rotating on its axis) or dynamic eccentricity (rotor rotating on the stator bore axis but not on its own axis). Obviously both types of eccentricity can exist together and in [2], [11] the eccentricity was studied (with finite element analysis (FEA)) in general terms of a rotating eccentricity which does not correspond to rotor speed. There is small level of static eccentricity existing even in newly manufactured machines due to the method of manufacturing and assembly. It can result in steady unbalanced onedirection UMP. During the run time, this may drive to bent rotor shaft, bearing wear and tear, etc. Furthermore, static eccentricity can become a reason for a certain degree of dynamic eccentricity. If these effects are not detected at early stage they can lead to rotor rub, which, in turn, will cause major breakdown of the machine.

In this paper a mathematical analysis is put forward in terms of static rotor eccentricity which varies down from the axial length of the machine. If a machine with $p_{m}$ pole-pairs has search coils with $p_{m} \pm 1$ pole pairs then the analysis goes on to illustrate how the electro-motive forces (EMFs) induced into these coils can be calculated. The paper then gives some experimental results using a 4 pole wound rotor induction machine rig designed for measuring UMP. This is not straightforward to measure although [12] shows a method. The machine is modified with additional 2 pole and 6 pole windings. Some search winding voltages and UMP results are given. Pole-specific detection windings were outlined in [13]. This concept is developed further here.

\section{A. Condition Monitoring Techniques Review}

Condition monitoring of induction motors has been a challenging task for the engineers and researchers although there are now several commercial systems available to industry; they are mostly aimed at the monitoring of cage induction motors. The aim is to achieve a relatively low cost and/or non-invasive system which is still powerful in terms of monitoring for online detection of developing faults. Many plants are of high cost capital investment or high turn-over production so that it is very advantageous to invest in a monitoring system. With a condition monitoring system, a fault can be detected at an early stage. Maintenance can then be carried out at planned downtime at an early stage, protecting the machine or plant from catastrophic failures and reducing the cost of maintenance. There are many diagnostic methods of detecting faults in induction machines, depending on the method of monitoring.

One of the most popular methods for fault diagnosis is the motor current signature analysis (MCSA) as it is more 
practical and less costly than others. The motor fault diagnosis can be done in real-time by analyzing frequency components of stator current signals. This was discussed in [14], [15] and this system allows precise and low-cost motor fault detection and it is non-invasive. Focusing on eccentricity faults detection, the MCSA methods developed are couched in terms of cage induction machines which may not be applicable to wound rotor machines as studied here.

Acoustic noise generated by air gap eccentricity in induction motors can be used for fault detection. Noise monitoring is carried out by measuring and analyzing the acoustic noise spectrum. In [16], Ellison and Yang carried out tests in an anechoic chamber. It was found that slot harmonics in the acoustic noise spectra from an induction motor were functions of static eccentricity.

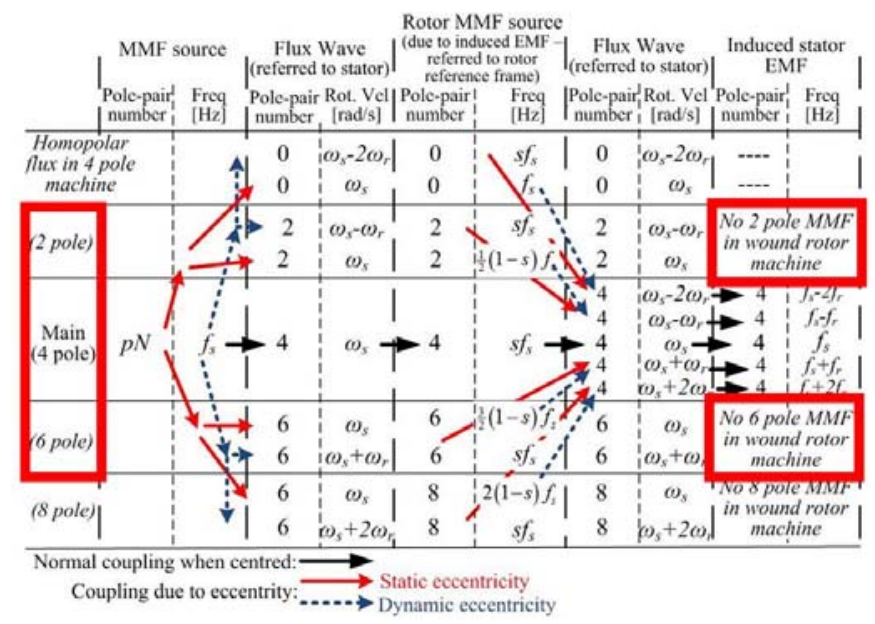

Fig. 1. Airgap MMF and flux waves in terms of pole or harmonics number and frequency.

Extensive work has been done in the detection of motor faults by monitoring vibration. Cameron, Thomson and Dow [17] verified that air gap eccentricity has resulted in vibratory harmonics at frequencies of $\left(f_{m}, f_{m 2}, f_{m 3}\right.$, or $\left.f_{m 4}\right)$. However, this method requires expensive sensors or specialized tools fitted to a specific location on the machine which weakens this technique; line current monitoring only requires Rogowski coils fitted to supply lines at the terminal box. Beguenane and Benbouzid [18] carried out thermal monitoring technique for induction motor using rotor resistance measurement. Another system was described in [19]. Many methods were reviewed in [20], [21].

A flux monitoring scheme can give reliable and accurate information about electrical machine conditions and such a system was described in [22]. If there is a change in the air gap flux or winding voltage, this can indicate a developing fault and it can be reflected in the harmonic spectrum. Verma and Natarajan [23] reported the change of air gap flux density as a function of static eccentricity. Thomson, Rankin, and Dorrell [24][25] analyzed the respective relationships among air gap flux, stator current, vibration signal, and air gap eccentricity by strict analytical equations. [26] presented a method for condition monitoring of electrical drives using special flux sensors. Rotor bar faults as well as eccentricity were determined based on leakage flux measurement [27], which is obtained by means of a search coil placed at the rear end of the machine. The leakage flux measurement is low cost, less affected by other magnetic circuits in the industrial environment, and easy to implement. [28] designed search coils which are placed under the stator winding wedges of the motor, and they were used for measuring the actual magnetic flux.

\section{B. Presented Work}

To summarize, this paper brings together work in [7], which put forward the concept of using damper windings to suppress UMP, and in [12], which presented an experimental study on a constructed rig, that can be used to measure UMP. The work develops the idea of using pole-specific windings in order to detect rotor eccentricity. Initial results were put forward in [29]. Here, an impedance matrix is developed to predict the winding voltages as a function of eccentricity. This is similar to that developed in [30]; however, it is couched in terms of a wound rotor induction motor rather than a cage machine. The primary motivation of this work was to move the auxiliary windings technique one step forward for control purpose. The newly investigated design for search coils will be put forward to control the UMP.

\section{ANALYSIS}

The aim of the work is to investigate the effectiveness of detecting rotor eccentricity using pole-specific windings. First, the airgap length needs to be described in terms of the circumferential distance around the airgap ( $y$ direction) and axial length down from the stator bore ( $x$ direction). This is shown in Fig. 2 and described below. With this model the induced EMF in pole-specific search coils need to be calculated. To simplify the approach, only the static eccentricity is studied here.

\section{A. Non-uniform Static Eccentricity}

If the rotor exhibits static eccentricity, the air-gap length can be expressed as varying in the circumferential $(y)$ direction and axial $(x)$ direction where

$$
\begin{aligned}
g_{s}(x, y) & =g(1-d(x) \cos (k y-\phi(x))) \\
& =g\left(1-\frac{d(x)}{2}\left(e^{(k y-\phi(x))}+e^{-(k y-\phi(x))}\right)\right) \\
& =g\left(1-0.5\left(\begin{array}{l}
d_{D E}(x) e^{j\left(k y-\phi_{D E}\right)} \\
+d_{N D E}(x) e^{j\left(k y-\phi_{N D E}\right)} \\
+d_{D E}(x) e^{-j\left(k y-\phi_{D E}\right)} \\
+d_{N D E}(x) e^{-j\left(k y-\phi_{N D E}\right)}
\end{array}\right)\right)
\end{aligned}
$$

This needs to be inverted to obtain the permeance waves for the airgap length: 


$$
\begin{aligned}
\Lambda_{s}(x, y) & =1 / g_{s}(x, y) \\
& =1 / g\left(1+\sum_{m=-\infty}^{\infty} \lambda^{m}(x) \cos (k y-\phi(x))\right) \\
& \approx 1 / g\left(1+\bar{\delta}(x)\left(e^{j(k y-\phi(x))}-e^{-j(k y-\phi(x))}\right)\right) \\
& =1 / g\left(1+\left(\begin{array}{l}
\delta_{D E}(x) e^{j\left(k y-\phi_{D E}\right)} \\
+\delta_{N D E}(x) e^{j\left(k y-\phi_{N D E}\right)} \\
+\delta_{D E}(x) e^{-j\left(k y-\phi_{D E}\right)} \\
+\delta_{N D E}(x) e^{-j\left(k y-\phi_{N D E}\right)}
\end{array}\right)\right)
\end{aligned}
$$

The analysis given above assumes that the eccentricity is not high so that the average permeance is the inverse of the airgap length. The coefficients can be defined by

$$
\begin{aligned}
\delta_{D E}(x) & =\left(\left(L_{s t}+2 x\right) / 2 d_{D E} L_{s t}\right) \\
\text { and } \quad \delta_{N D E}(x) & =\left(\left(L_{s t}-2 x\right) / 2 d_{N D E} L_{s t}\right)
\end{aligned}
$$

Assuming that $x$ is defined as zero at the centre of the bore in the axial direction and the $D E$ eccentricity decreases to zero when moving from the $D E$ to the $N D E$. Conversely the $N D E$ eccentricity decreases from the $N D E$ to the $D E$. This is illustrated in Fig. 2. $L_{s t}$ is the axial length.

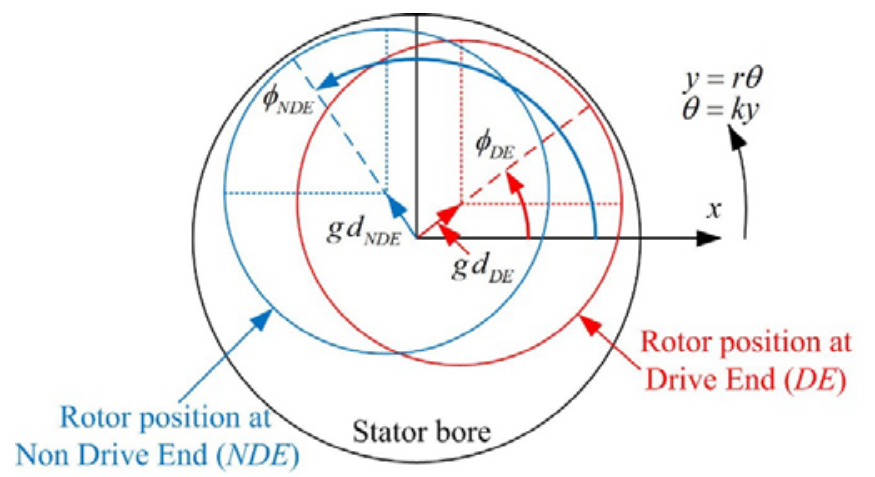

Fig. 2. Definition of $D E$ and $N D E$ eccentricity.

\section{B. Generation of Additional Airgap Flux Waves}

If the 3-phase supply is balanced with series connected stator windings, the MMF wave is

$$
j_{s t}(y, t)=\operatorname{Re} \sum_{n=-\infty}^{\infty} \bar{J}_{s t}^{n} e^{j\left(\omega t-n p_{m} k y\right)}
$$

where, for a balance 3-phase current set, and using the identity $a=\exp (j 2 \pi / 3)$, the MMF magnitude is

$$
\bar{J}_{s t}^{n}=\bar{N}_{s t}^{n}\left(1+a^{1-n} a^{n-1}+a^{n-1} a^{1-n}\right) \bar{I}_{s}=\left.3 \bar{N}_{s t}^{n} \bar{I}_{s}\right|_{n=1,-5,7 \ldots}
$$

and the fundamental pole-pair number of the machine is $p_{m}$. This includes winding harmonics $n . I_{s}$ is the phase current phasor for phase $a$. This is a correction to the equation as denoted in [6], [7]. The stator winding coefficient of one phase winding is

$$
\bar{N}_{s t}^{n}=\frac{k}{2 \pi} \sum_{w=1}^{N_{w}} k_{s} C_{w} e^{j n p_{m} k y_{w}}
$$

where $N_{w}$ is the number of slots that the winding is located in, $C_{w}$ is the number of series winding turns in a slot, and $y_{w}$ is the spatial location of the slot. The slot opening factor is defined by the slot opening $b_{s}$ (in $\mathrm{m}$ ) where

$$
k_{s}=\frac{2 \sin \left(0.5 n p_{m} k b_{s}\right)}{n p_{m} k b_{s}}
$$

Ampere's circuital law can be applied using

$$
b_{s t}(x, y, t)=\mu_{0} \Lambda_{s}(x, y)\left[\int j_{s t}(y, t) d y+C\right]
$$

The constant $C$ is the homopolar flux term control as described in [30] and [31]. This is particularly relevant to a 2 pole machine where $p_{m}-1=0$ which is a homopolar flux that crosses the airgap once and finds a return path via some other route through the bearings and end region. This leads to a distribution of the air-gap flux density due to the stator MMF waves:

$$
b_{s t}(x, y, t)=\operatorname{Re} \sum_{n=-\infty}^{\infty}\left[\begin{array}{l}
\bar{B}_{s t}^{n p_{m}} e^{j\left(\omega t-n p_{m} k y\right)} \\
+\bar{B}_{s t}^{n p_{m}-1}(x) e^{j\left(\omega t-k\left(n p_{m}-1\right) y\right)} \\
+\bar{B}_{s t}^{n p_{m}+1}(x) e^{j\left(\omega t-k\left(n p_{m}+1\right) y\right)}
\end{array}\right]
$$

The field magnitudes are

$$
\bar{B}_{s t}^{n p_{m}}=\frac{j \mu_{0} \bar{J}_{s t}^{n}}{k n p_{m} g}, \text { and } \bar{B}_{s t}^{n p_{m} \pm 1}(x)=\frac{j \mu_{0} \bar{J}_{s t}^{n}}{k n p_{m} g} \bar{\delta}(x)
$$

This is for a machine with more than 2 poles. Refer to [30] and [31] for the special case of a 2 pole machine.

\section{Eccentricity Detection}

Once the terms for the additional airgap flux waves have been obtained then the UMP can be calculated as illustrated in [6]. However, to get the EMF induced in the pole-specific search coils, we need the airgap electric field which can be obtained from:

$$
e^{p}(x, y, t)=\frac{\mathrm{d} b^{p}(x, y, t)}{\mathrm{d} t} \mathrm{~d} y=\bar{E}_{s t}^{p_{m} \pm 1}(x) e^{j\left(\omega t-k\left(p_{m} \pm 1\right) y\right)}
$$

where

$$
\bar{E}_{s t}^{p_{m} \pm 1}(x)=\frac{-\bar{B}_{s t}^{n p_{m} \pm 1}(x)}{k\left(p_{m} \pm 1\right)}=\frac{-j \mu_{0} \omega \bar{J}_{s t}^{n}}{k^{2} p_{m}\left(p_{m} \pm 1\right) g} \bar{\delta}(x)
$$

For a machine with $p_{m}$ pole-pairs, it can be assumed that there are two pole-specific search windings with pole-pair numbers $p_{m} \pm 1$. Each pole-pair has two windings in quadrature on $\alpha$ and $\beta$ axes. The EMFs induced in these windings can be obtained from:

$$
\begin{aligned}
& u_{(S C+1)_{(\alpha, \beta)}}(t)=\operatorname{Re} \int_{-L_{s s} / 2}^{L_{s s} / 2} \int_{0}^{2 \pi r}-e^{p_{m}+1}(x, y, t) n_{(S C+1)_{(\alpha, \beta)}}^{P_{p+1}}(y) \mathrm{d} y \mathrm{~d} x \\
& u_{(S C-1)_{(\alpha, \beta)}}(t)=\operatorname{Re} \int_{-L_{s / 2} / 2}^{L_{s} / 2} \int_{0}^{2 \pi r}-e^{p_{m}-1}(x, y, t) n_{(S C-1)_{(\alpha, \beta)}}^{P_{m}-1}(y) \mathrm{d} y \mathrm{~d} x
\end{aligned}
$$


where the search winding coefficients are, for the $p_{m}+1$ polepairs and for $p_{m}-1$ pairs:

$$
\begin{aligned}
& n_{(S C+1)_{\alpha}}^{p_{m}+1}(y)=\left(\bar{N}_{(S C+1)}^{p_{m}+1}\right)^{*} e^{-j(p+1) k y} \\
& n_{(S C+1)_{\beta}}^{p_{m}+1}(y)=\left(j \bar{N}_{(S C+1)}^{p_{m}+1}\right)^{*} e^{-j(p+1) k y} \\
& n_{(S C-1)_{\alpha}}^{p_{m}-1}(y)=\left(\bar{N}_{(S C-1)}^{p_{m}-1}\right)^{*} e^{-j(p-1) k y} \\
& n_{(S C-1)_{\beta}}^{p_{m}-1}(y)=\left(j \bar{N}_{(S C-1)}^{p_{m}-1}\right)^{*} e^{-j(p-1) k y}
\end{aligned}
$$

where

$$
\begin{aligned}
\bar{N}_{S C+1}^{p_{m}+1} & =\frac{k}{2 \pi} \sum_{w=1}^{N_{w}} k_{s} C_{w(S C+1)} e^{j n\left(p_{m}+1\right) k y_{w}} \\
\text { and } \bar{N}_{S C-1}^{p_{m}-1} & =\frac{k}{2 \pi} \sum_{w=1}^{N_{w}} k_{S} C_{w(S C-1)} e^{j n\left(p_{m}-1\right) k y_{w}}
\end{aligned}
$$

For simplicity, it is assumed that the $\alpha$ winding is on the real axis and the $\beta$ winding is on the imaginary axis. Phase $a$ of the main winding is also located on the real axis. This is the expression for the voltage induced in the search coil by a rotating flux wave set up by a balanced 3-phase current. If the current set is unbalanced, then a backward rotating flux wave will be set up and this can be accounted for and will be the focus of further work. In [6] static eccentricity tends to produce a steady pull in one direction, while dynamic eccentricity creates a UMP vector rotating in synchronism with the rotor. The analysis model of the dynamic eccentric can be derived in the same way of static eccentricity.

If the winding harmonics are ignored so $n=1$ only, then the airgap flux is approximately:

$$
b_{s t}(x, y, t) \approx\left(\begin{array}{l}
\bar{B}_{s t}^{p_{m}} e^{j\left(\omega t-p_{m} k y\right)} \\
+\bar{B}_{s t}^{p_{m}-1}(x) e^{j\left(\omega_{1} t-k\left(p_{m}-1\right) y\right)} \\
+\bar{B}_{s t}^{p_{m}+1}(x) e^{j\left(\omega_{2} t-k\left(p_{m}+1\right) y\right)}
\end{array}\right)
$$

where

$$
\begin{aligned}
\bar{B}_{s t}^{p_{m} \pm 1}(x) & =\frac{j \mu_{0} \bar{J}_{s t}}{k p_{m} g} \bar{\delta}(x) \\
& =\frac{j \mu_{0} \bar{J}_{s t}}{k p_{m} g}\left(\delta_{D E}(x) e^{\mp\left(\phi_{D E}\right)}+\delta_{N D E}(x) e^{\mp\left(\phi_{N D E}\right)}\right)
\end{aligned}
$$

For the static eccentricity

$\omega_{1}=\omega_{2}=\omega \quad$ (i.e., a constant radial force in that direction)

For the dynamic eccentricity

$\omega_{1}=\omega-\omega_{r}$, and $\omega_{2}=\omega+\omega_{r}$. (i.e., there is a rotational frequency radial vibration which corresponds to a rotating force vector).

The flux waves have to be transformed into the rotor reference frame in order to calculate the EMFs induced into the cage and hence obtain the rotor current and MMF. For a spatial circumferential position $y^{\prime}$ on the rotor: $k y=\omega_{r} t+k y^{\prime}$.

The study in [6] indicated that both static and dynamic eccentricity produces their own $p_{m}^{ \pm 1}$ field components, the only difference between them being the rotational velocity. Also the EMF induced into the additional windings is influenced by the air-gap flux density. So the pole-specific search coil method absolutely could be used to predict the dynamic eccentricity. This will be presented in the new work after developing the test rig.

Obviously as the machine is loaded this approximation becomes less valid but it can be used to calculate the search winding voltages. The other flux waves due to higher winding harmonics can be treated as leakage flux.

\section{Impedance Matrix}

An impedance matrix can be developed that incorporates eccentricity and also the pole-specific search windings. As developed in [30] and [31] it is possible to develop a coupling impedance matrix.

\section{1) Condition monitoring}

If we focus on the condition monitoring function of the search coils when the rotor is open circuit, then:

$$
\left[\begin{array}{l}
v_{s-p h} \\
v_{p m-1}^{\alpha} \\
v_{p m-1}^{\beta} \\
v_{p m+1}^{\alpha} \\
v_{p m+1}^{\beta}
\end{array}\right]=[\bar{Z}]\left[\begin{array}{l}
i_{s-p h} \\
0 \\
0 \\
0 \\
0
\end{array}\right]=\left[\begin{array}{ccccc}
\bar{Z}_{s, s} & 0 & 0 & 0 & 0 \\
\bar{Z}_{p m-1, s}^{\alpha} & 0 & 0 & 0 & 0 \\
\bar{Z}_{p m-1, s}^{\beta} & 0 & 0 & 0 & 0 \\
\bar{Z}_{p m+1, s}^{\alpha} & 0 & 0 & 0 & 0 \\
\bar{Z}_{p m+1, s}^{\beta} & 0 & 0 & 0 & 0
\end{array}\right]\left[\begin{array}{l}
i_{s-p h} \\
0 \\
0 \\
0 \\
0
\end{array}\right]
$$

where

$$
\begin{aligned}
\bar{Z}_{s, s} & =R_{p h}+j X_{\text {leakage }}+j X_{\text {mag }} \\
\bar{Z}_{p m-1, s}^{\alpha} & \left.=j \frac{3 \pi \mu_{0} \omega L_{s t} \bar{N}_{s t}^{1}}{k^{3} p_{m}\left(p_{m}-1\right) g} \bar{\delta} \text { (mean }\right)\left(\bar{N}_{(S C-1)}^{p_{m}-1}\right)^{*} \\
\bar{Z}_{p m-1, s}^{\beta} & =j \frac{3 \pi \mu_{0} \omega L_{s t} \bar{N}_{s t}^{1}}{k^{3} p_{m}\left(p_{m}-1\right) g} \bar{\delta} \text { (mean) }\left(j \bar{N}_{(S C-1)}^{p_{m}-1}\right)^{*} \\
X_{\text {mag }} & =\frac{3 \pi \mu_{0} \omega L_{s t} \bar{N}_{s t}^{1}}{k^{3} p_{m}^{2} g}\left(\bar{N}_{s t}^{1}\right)^{*}
\end{aligned}
$$

Similar expressions exist for the $\left(p_{m}+1\right)$ linkage impedances. This is the most basic form of this impedance matrix. The leakage reactance includes all the end effects, slot leakage and winding harmonics. It can be further developed to include unbalanced currents as included in [30].

\section{2) UMP control}

The matrix can be further developed to include additional winding current and active UMP control. If passive damping is used, i.e., the windings are short circuited, then 


$$
\left[\begin{array}{l}
v_{s-p h} \\
0 \\
0 \\
0 \\
0
\end{array}\right]=\left[\begin{array}{lllll}
\bar{Z}_{s, s} & \bar{Z}_{s, p m-1}^{\alpha} & \bar{Z}_{s, p m-1}^{\beta} & \bar{Z}_{s, p m+1}^{\alpha} & \bar{Z}_{s, p m+1}^{\beta} \\
\bar{Z}_{p m-1, s}^{\alpha} & \bar{Z}_{p m-1, C S}^{\alpha} & 0 & 0 & 0 \\
\bar{Z}_{p m-1, s}^{\beta} & 0 & \bar{Z}_{p m-1, C S}^{\beta} & 0 & 0 \\
\bar{Z}_{p m+1, s}^{\alpha} & 0 & 0 & \bar{Z}_{p m+1, C S}^{\alpha} & 0 \\
\bar{Z}_{p m+1, s}^{\beta} & 0 & 0 & 0 & \bar{Z}_{p m+1, C S}^{\beta}
\end{array}\right]\left[\begin{array}{c}
i_{s-p h} \\
i_{p m-1}^{\alpha} \\
i_{p m-1}^{\beta} \\
i_{p m+1}^{\alpha} \\
i_{p m+1}^{\beta}
\end{array}\right]
$$

where

$$
\begin{aligned}
\bar{Z}_{s, p m-1}^{\alpha} & =j \frac{\pi \mu_{0} \omega L_{s t} \bar{N}_{s t}^{-1}}{k^{3} p_{m}\left(p_{m}-1\right) g} \bar{\delta} \text { (mean) }\left(\bar{N}_{(S C-1)}^{p_{m}-1}\right) \\
\bar{Z}_{s, p m-1}^{\beta} & \left.=j \frac{\pi \mu_{0} \omega L_{s t} \bar{N}_{s t}^{-1}}{k^{3} p_{m}\left(p_{m}-1\right) g} \bar{\delta} \text { (mean) }\right)\left(j \bar{N}_{(S C-1)}^{p_{m}-1}\right) \\
\bar{Z}_{p m-1, S C}^{\alpha} & =R_{S C-1}+j \frac{\pi \mu_{0} \omega L_{s t} \bar{N}_{(S C-1)}^{p_{m}-1}}{k^{3}\left(p_{m}-1\right)^{2} g}\left(\bar{N}_{(S C-1)}^{p_{m}-1}\right)^{*} \\
\bar{Z}_{p m-1, S C}^{\beta} & =R_{S C-1}+j \frac{\pi \mu_{0} \omega L_{s t} \bar{N}_{(S C-1)}^{p_{m}-1}}{k^{3}\left(p_{m}-1\right)^{2} g}\left(j \bar{N}_{(S C-1)}^{p_{m}-1}\right)^{*}
\end{aligned}
$$

and $R_{s c-I}$ is 2 pole winding resistance. The self-inductances of the 2-pole and 6-pole windings are almost constant. Also the mutual inductances between the two windings of each winding set are zero, because these windings are 2-phase and wound in quadrature, and there are no speed terms. Developing the UMP detection matrix could be done to include the rotor current as

$$
\left[\begin{array}{l}
v_{s-p h} \\
v_{r-p h} \\
v_{p m-1}^{\alpha} \\
v_{p m-1}^{\beta} \\
v_{p m+1}^{\alpha} \\
v_{p m+1}^{\beta}
\end{array}\right]=[\bar{Z}]\left[\begin{array}{l}
i_{s-p h} \\
i_{r-p h} \\
0 \\
0 \\
0 \\
0
\end{array}\right]=\left[\begin{array}{llllll}
\bar{Z}_{s, s} & \bar{Z}_{s, r} & 0 & 0 & 0 & 0 \\
\bar{Z}_{r, s} & \bar{Z}_{r, r} & 0 & 0 & 0 & 0 \\
\bar{Z}_{p m-1, s}^{\alpha} & \bar{Z}_{p m-1, r}^{a} & 0 & 0 & 0 & 0 \\
\bar{Z}_{p m-1, s}^{\beta} & \bar{Z}_{p m-1, r}^{\beta} & 0 & 0 & 0 & 0 \\
\bar{Z}_{p m+1, s}^{\alpha} & \bar{Z}_{p m+1, r}^{a} & 0 & 0 & 0 & 0 \\
\bar{Z}_{p m+1, s}^{\beta} & \bar{Z}_{p m+1, r}^{\beta} & 0 & 0 & 0 & 0
\end{array}\right]\left[\begin{array}{l}
i_{s-p h} \\
i_{r-p h} \\
0 \\
0 \\
0 \\
0
\end{array}\right]
$$

And similar linkage impedances exist for $p_{m}+1$. We can obtain the UMP as given in [7] where

$$
\left[\begin{array}{l}
F_{\alpha} \\
F_{\beta}
\end{array}\right]=\left[\begin{array}{lllll}
F_{\alpha, p h} & F_{\alpha, p m-1}^{\alpha} & F_{\alpha, p m-1}^{\beta} & F_{\alpha, p m+1}^{\alpha} & F_{\alpha, p m+1}^{\beta} \\
F_{\beta, p h} & F_{\beta, p m-1}^{\alpha} & F_{\beta, p m-1}^{\beta} & F_{\beta, p m+1}^{\alpha} & F_{\beta, p m+1}^{\beta}
\end{array}\right]\left[\begin{array}{c}
i_{p h} \\
i_{p m-1}^{\alpha} \\
i_{p m-1}^{\beta} \\
i_{p m+1}^{\alpha} \\
i_{p m+1}^{\beta}
\end{array}\right]
$$

Equation matrices (20) and (23) can be combined and active voltage control used for UMP reduction as in (24); this can be programmed and will be the focus of further study.

$$
\left[\begin{array}{c}
v_{s-p h} \\
v_{p m-1}^{\alpha} \\
v_{p m-1}^{\beta} \\
v_{p m+1}^{\alpha} \\
v_{p m+1}^{\beta} \\
F_{\alpha}^{\alpha} \\
F_{\beta}
\end{array}\right]=\left[\begin{array}{lllll}
\bar{Z}_{s, s} & \bar{Z}_{s, p m-1}^{\alpha} & \bar{Z}_{s, p m-1}^{\beta} & \bar{Z}_{s, p m+1}^{\alpha} & \bar{Z}_{s, p m+1}^{\beta} \\
\bar{Z}_{p m-1, s}^{\alpha} & \bar{Z}_{p m-1, C S}^{\alpha} & 0 & 0 & 0 \\
\bar{Z}_{p m-1, s}^{\beta} & 0 & \bar{Z}_{p m-1, C S}^{\beta} & 0 & 0 \\
\bar{Z}_{p m+1, s}^{\alpha} & 0 & 0 & \bar{Z}_{p m+1, C S}^{\alpha} & 0 \\
\bar{Z}_{p m+1, s}^{\beta} & 0 & 0 & 0 & \bar{Z}_{p m+1, C S}^{\beta} \\
F_{\alpha, p h} & F_{\alpha, p m-1}^{\alpha} & F_{\alpha, p m-1}^{\beta} & F_{\alpha, p m+1}^{\alpha} & F_{\alpha, p m+1}^{\beta} \\
F_{\beta, p h} & F_{\beta, p m-1}^{\alpha} & F_{\beta, p m-1}^{\beta} & F_{\beta, p m+1}^{\alpha} & F_{\beta, p m+1}^{\beta}
\end{array}\right]\left[\begin{array}{c}
i_{s-p h} \\
i_{p m-1}^{\alpha} \\
i_{p m-1}^{\beta} \\
i_{p m+1}^{\alpha} \\
i_{p m+1}^{\beta}
\end{array}\right]
$$

\begin{tabular}{|c|c|}
\hline \multicolumn{2}{|c|}{$\begin{array}{c}\text { TABLE I } \\
\text { MACHINE SPECIFICATION }\end{array}$} \\
\hline \multicolumn{2}{|c|}{ Name plate details } \\
\hline Power [HP] & $10($ or $7.46 \mathrm{~kW})$ \\
\hline Speed $[\mathrm{rpm}]$ & 1420 \\
\hline Frequency $[\mathrm{Hz}]$ & 50 \\
\hline Stator voltage $[\mathrm{V}]$ & 400/440 Delta \\
\hline Stator rated current $[\mathrm{A}]$ & 13 \\
\hline Rotor Voltage [V] & 200 \\
\hline Rated rotor current $[\mathrm{A}]$ & 22 \\
\hline Number of Poles & 4 \\
\hline Slip [p.u.] & 0.0533 \\
\hline \multicolumn{2}{|c|}{ Measured geometry [mm] } \\
\hline Axial length rotor core & 103.9 \\
\hline Axial length stator core & 100.9 \\
\hline Stator outer diameter & 353 \\
\hline Stator inner diameter & 228.15 \\
\hline Rotor outer diameter & 226.42 \\
\hline Shaft diameter & 120 \\
\hline Airgap length & 0.5 \\
\hline Stator slots & 48 \\
\hline Stator slot opening & 3.9 \\
\hline Turns per slot & 34 in series \\
\hline Stator wire diameter & 1.725 - by calculation from $S P E E D$ \\
\hline Stator winding & Single layer lap with 13 slot pitch \\
\hline Coils per pole per phase & 2 \\
\hline Stator slot depth & 26.82 \\
\hline Stator tooth width & 8.22 \\
\hline Rotor slot opening & 3 \\
\hline Rotor slot depth & 22.5 \\
\hline Slot width & 3.5 \\
\hline Rotor wire diameter & $1.9 \mathrm{~mm}$ from $S P E E D$ \\
\hline Turns per coil & 6 in series \\
\hline Coils per phase & 12 \\
\hline Rotor connection & Star through 3 slip rings \\
\hline \multicolumn{2}{|c|}{ Measured resistances } \\
\hline Rph stator (DC, cold) & $1.82 \mathrm{ohm}$ \\
\hline Rph rotor (DC, cold) & $0.23 \mathrm{ohm}$ \\
\hline
\end{tabular}

\section{EXPERIMENTAL RIG AND RESULTS}

A 4 pole wound rotor induction machine was modified as shown in Fig. 3. Data for this machine are given in Table I and some data were obtained from simulating the machine in SPEED software [32].

The rotor for this machine was separately mounted on pedestals that included piezoelectric force transducers; these allowed the direct measurement of the UMP on each bearing. As the start of the study, the machine was modified to include the simple pole-specific search windings as illustrated in Fig. 3(a). These had 6 poles and 2 poles. Fig. 3(b) shows the 
mounting of the rotor with the transducers as well as the rotor slip-rings. Fig. 3(c) shows the clock dials that were used to measure the eccentricity measurement.

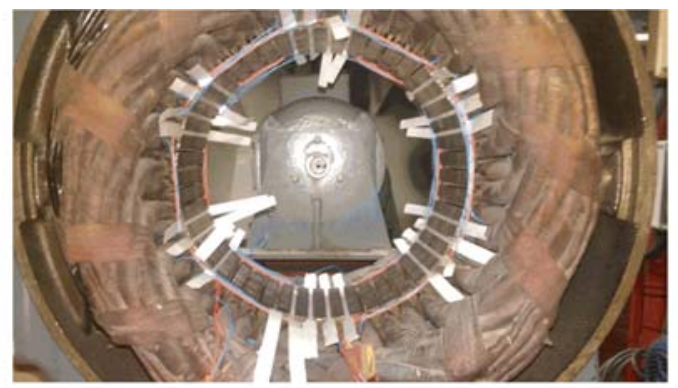

(a) Search coils being fitted: single 6 pole winding; red coils down top of slots; and blue coils wound round winding only next to end of core

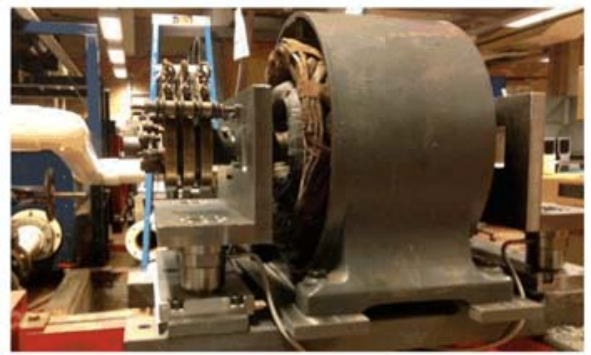

(b) Rig including UMP measurement

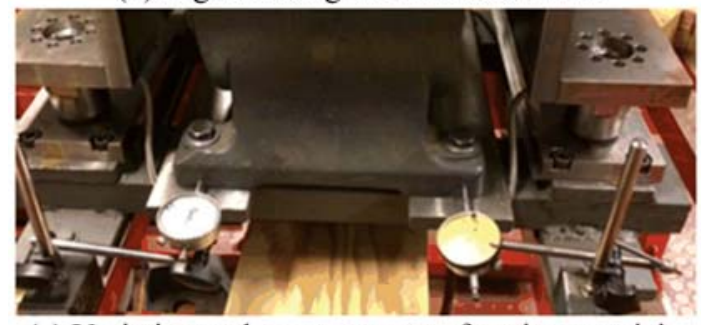

(c) Variation and measurement of static ecentricity

Fig. 3. Experimental with 4 pole wound rotor machine and additional 2 and 6 pole search windings. Clock guages (c) were used to measure the eccentricity which was set by moving the stator with respect to the rotor.

\section{A. Simple Search Coils}

The main winding was originally held in by slot top wedges. These were removed and the space was used to insert search windings. The 6-pole search windings consisted of 24 coils with two turns per coil. The span of each coil was 8 slots and half of the slots had a 6-pole coils side in the slot top. There were two 2-pole windings in quadrature. These were formed from 12 coils of one turn and 24 slot pitch. Each slot had on 2-pole slot side in it. Thinner wedges were made and used to hold the search windings in as illustrated in Fig 3(a). Centering and setting the eccentricity requires careful adjustment. During testing, when the rotor was nominally centered there was about $4 \%$ eccentricity; when there was nominally $20 \%$ eccentric it was found to be $14 \%$; and when there was nominally $40 \%$ eccentricity it was found to be about $30 \%$ eccentric. This was obtained via measurement of the UMP and comparison to finite element analysis as illustrated in [29]. The machine was further modified with a stator rewound with the same 3-phase 4-pole winding and incorporation of the 2-pole and 6-pole quadrature search windings into the slots. This allowed more substantial search windings to be installed which could carry sufficient current to damp the UMP. The rewound machine is shown in Fig. 4. This also shows the measurement system.

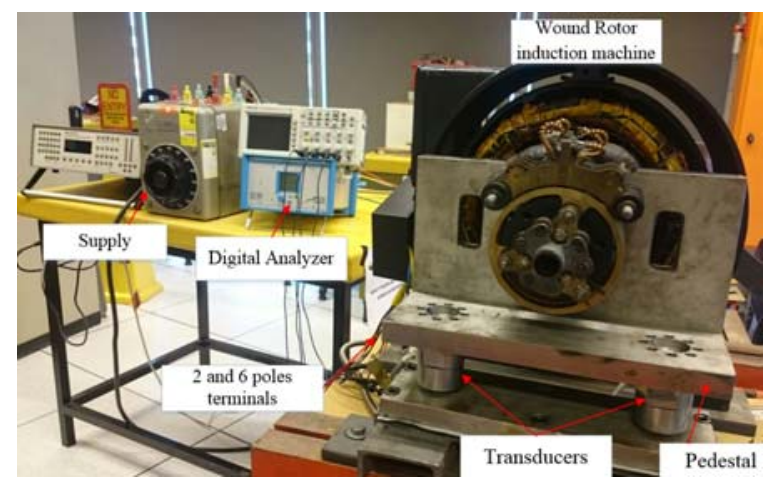

(a) System arrangement

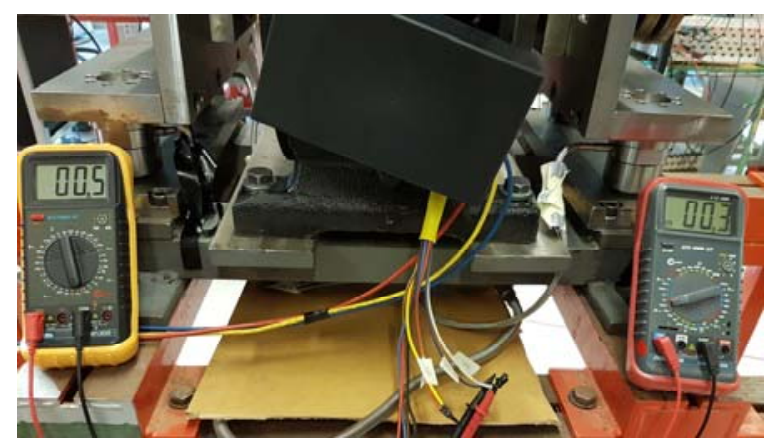

(b) Side view

Fig. 4. Instrumentation with rewound stator.

The first set of tests simply had one 6-pole search winding inserted. The results in Fig. 5(a) show an almost linear characteristic. The open-circuit (running light) and short circuit (locked rotor) results are different. The degree of eccentricity is not precise as discussed above and there is a degree of eccentricity variation down the axial length of the machine. The machine was then dismantled and two 2-pole search windings were inserted on quadrature axes ( $\alpha$ and $\beta$ ). The eccentricity was less in these tests but again there is a linear characteristic. The short circuit tests were done at $50 \mathrm{~V}$ and, if linearity is assumed, then these results should be four time less. However, an induction machine demagnetizes as it is loaded hence the results are less.

In Fig. 5(b) the 2 pole and 6 pole search winding voltages are given for open circuit operation and these show approximate linearity. These were initial results and the method of physically measuring the degree of eccentricity is still being developed. To test the theory developed above, the 
induced voltages in the winding was calculated for a given phase current and eccentricity. Equations were programmed in MATLAB.

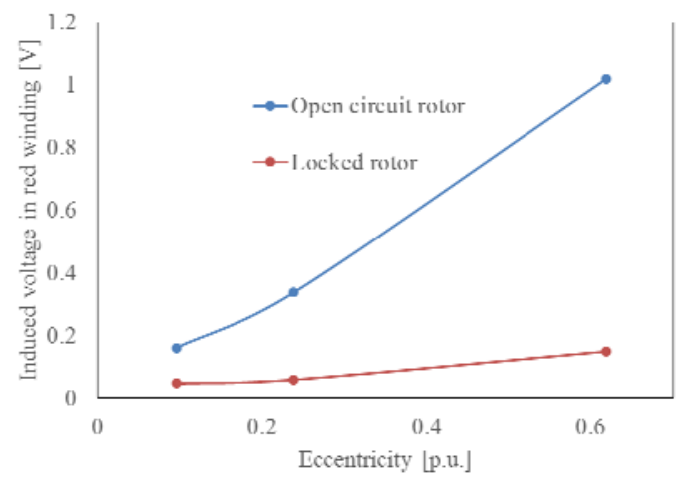

(a)

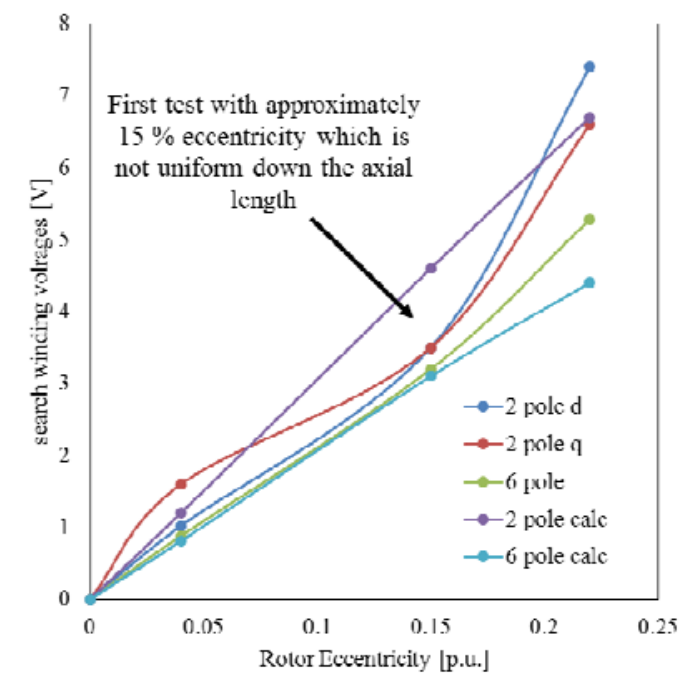

(b)

Fig. 5. Induced search winding voltages with a variation of eccentricity with 2 and 6 pole search windings.

It can be seen in Fig. 5(b) that the results match the measured reasonably well given the approximate nature of the eccentricity measurement. The search windings, if they are suitably robust with sufficient turns and conductor current carrying capacity, can be used to control the UMP (hence the later rewinding). This can be used to reduce bearing wear as described in [7].

\section{B. Functional Search Coils}

As discussed above, after some initial tests, the stator was rewound with the same 4-pole 3-phase winding but new six and two pole search windings were inserted; these were designed with more turns. They have two phases which were in quadrature. All the windings were correctly placed in the slots with suitable wedging. The winding schemes for the search windings are shown in Fig. 6 and the windings details are presented in Table II.
TABLE II

WINDING PARAMETERS

\begin{tabular}{|l|l|}
\hline \multicolumn{2}{|c|}{ Four-pole main winding } \\
\hline Phases & 3 \\
\hline Parallal paths & 1 \\
\hline Effective turns per slot & 34 \\
\hline Connection & Star \\
\hline Resistance (Room Temp.) & 1.4 Ohm \\
\hline \multicolumn{2}{|c|}{ Six-pole winding } \\
\hline Phases & 2 (in quadrature on $\alpha$ and $\beta$ axes) \\
\hline Parallal paths & 1 \\
\hline Effective turns per slot & 44 \\
\hline Connection & Short-Circuit \\
\hline Resistance (Room Temp.) & 22.5 Ohm \\
\hline \multicolumn{2}{|c|}{ Two-pole winding } \\
\hline Phases & 2 (in quadrature on $\alpha$ and $\beta$ axes) \\
\hline Parallal paths & 1 \\
\hline Effective turns per slot & 15 \\
\hline Connection & Short-Circuit \\
\hline Resistance (Room Temp.) & 14.3 Ohm \\
\hline
\end{tabular}
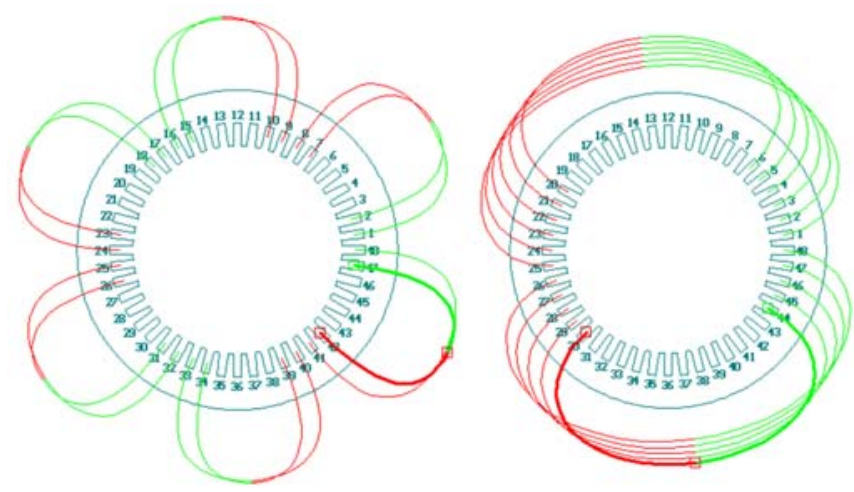

Fig. 6. 2-phase search windings: 6-pole search coils, 12 coils per phase, and 44 turns per coil and 2-pole search coils, 12 coils per phase, and 15 turns per coil - one phase of each shown.

In Fig. 7 the new search winding voltages are given for open circuit operation and locked rotor test, and these again show approximate linearity. The voltages induced into the 2 pole and 6-pole coils are nearly identical and this can be accounted for by addressing the total turns and flux linkage per pole. The total turns per phase for the 6-pole winding is 528 with a peak flux linkage of $\Phi_{6}$ so that the flux linkage is $528 \Phi_{6}$. From (9) and (10) the peak flux densities of the 2 pole and 6 pole flux waves and the frequencies are the same. Therefore, the flux linkage per pole for the 2 and 6-pole flux waves are approximately $\Phi_{2}=3 \Phi_{6}$. The total turns per phase for the 2-pole winding is 180 so that the flux linkage is $180 \Phi_{2}=540 \Phi_{6}$. Hence, with the same frequency, the induced open-circuit search winding voltages are almost equal.

The measured and calculated search coil voltages are compared in Fig. 8. This is with an open-circuit rotor. The voltages are now much higher than those with the first search coil arrangements due to the increased number of turns. The voltages are close and differences will be due to the variation between the nominal eccentricity setting and actual setting. The $5 \%$ eccentricity setting is probably close to $10 \%$ and the $40 \%$ eccentricity setting is probably close to $35 \%$ 
eccentricity. The 2 pole winding is measuring lower voltage because the machine is designed as a 4 pole machine so the stator yoke is narrower than an equivalent 2-pole machine; hence, there would be some finite MMF drop around the core-back and a lowering of the 2-pole flux. However, the results appear reasonable.

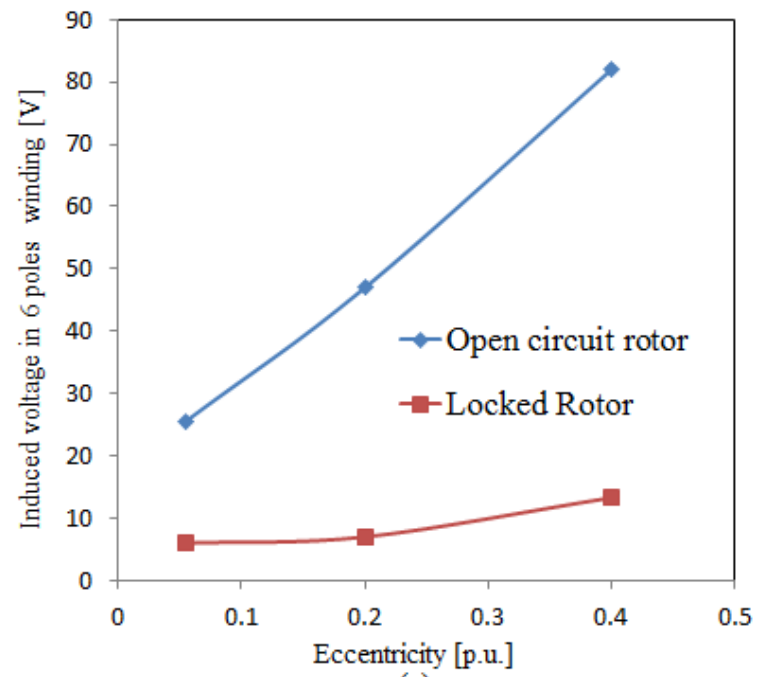

(a)

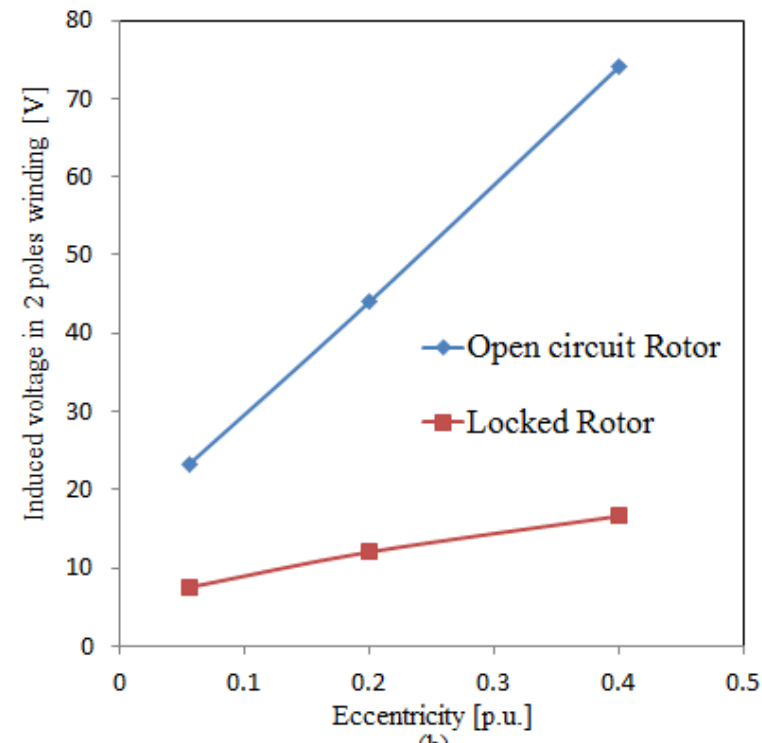

(b)

Fig. 7. Induced search winding voltages with a variation of eccentricity.

The search windings are now short circuited to produce effective damper windings. The measured and calculated damper currents are given in Fig. 9 and appear close given the issues with setting the degree of eccentricity. The currents increase rapidly with eccentricity level. The UMP characteristics are shown Fig. 10; these show the UMP results with the search coils winding open-circuited so there is no damping current. These results are compared the UMP result with the winding short-circuited. These illustrate that the damper windings significantly damp the UMP at the synchronous speed but the effect is much reduced when the rotor is locked. This is because as the slip increases the machine demagnetizes and the fundamental stator and rotor MMFs cancel each other. However, the winding harmonics begin to influence the UMP. This was discussed in [30] and [31]. The damping effect of the 2 and 6 pole windings are for the fundamental flux wave therefore it is to be expected that the UMP damping is much lower under locked rotor conditions. For a doubly-fed induction generator (DFIG), as often used in wind turbines, then they will operate between about $\mathrm{s}=0.25$ to $\mathrm{s}=-0.25$ [32] under controlled stator and rotor currents so that the damping windings will be effective under these conditions.

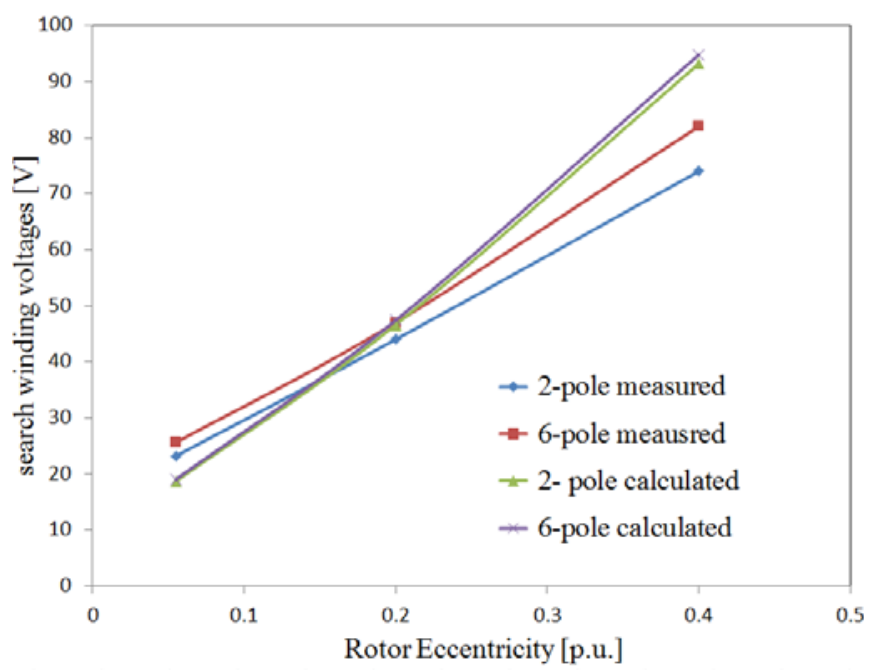

Fig. 8. Calculated and measured voltages in the search winding with a variation of eccentricity.

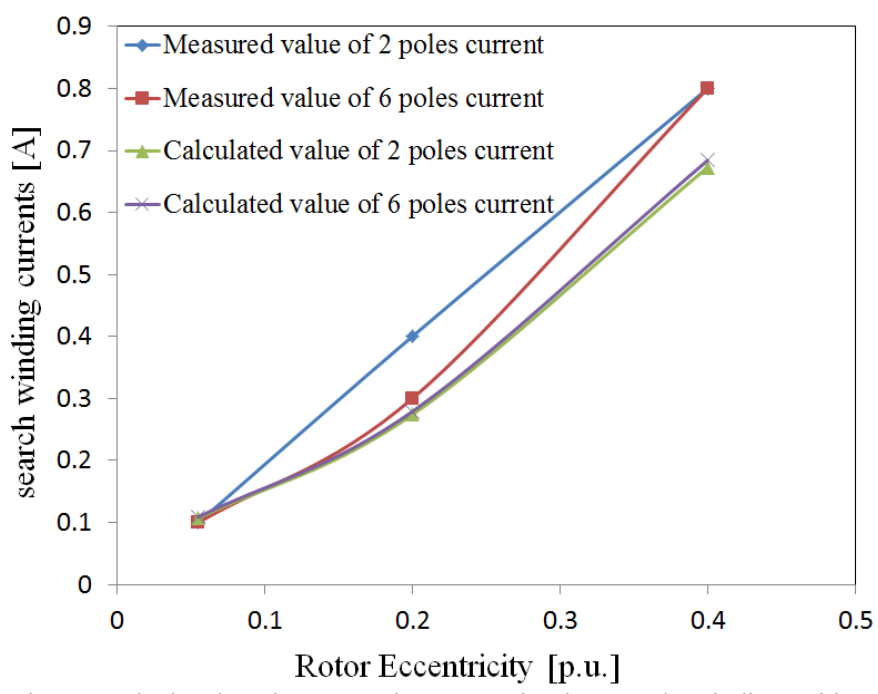

Fig. 9. Calculated and measured currents in the search winding with a variation of eccentricity.

The measured and calculated search coil losses are compared in Fig. 11. The losses in the 6-pole winding was more than the 2-pole because it has higher resistor than 2pole winding. Obviously, the losses are increasing with increase of the eccentricity level. It is worthy, using winding copper losses in detection the eccentricity failure. Using the 
matrices in (18) and (20), UMP could be calculated as in Fig. 12.

To evaluate the effectiveness of the Auxiliary Windings method, the vibration was sensed on the stator frame and on the bearing housing. The vibration spectra are presented in Fig. 13. The results exhibited that the amplitude of principal harmonic in the motor was reduced after shorted the additional windings. The black circle in the figure was used to show the reduction in the amplitude.

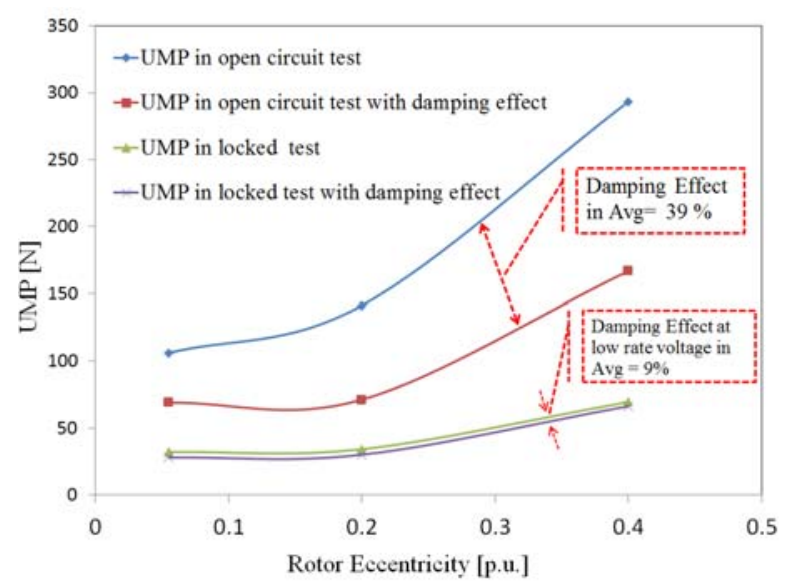

Fig. 10. Variation of measured UMP with eccentricity at open circuit test and locked rotor test.

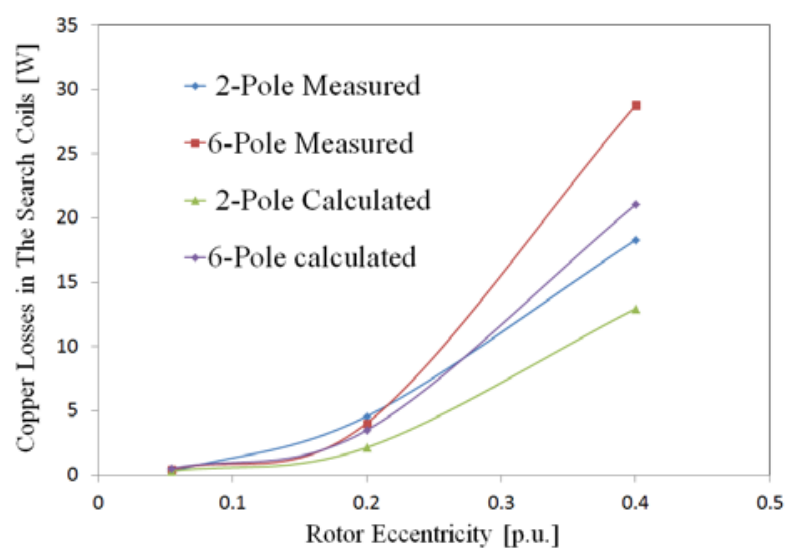

Fig. 11. Calculated and measured copper losses in the search winding with a variation of eccentricity.

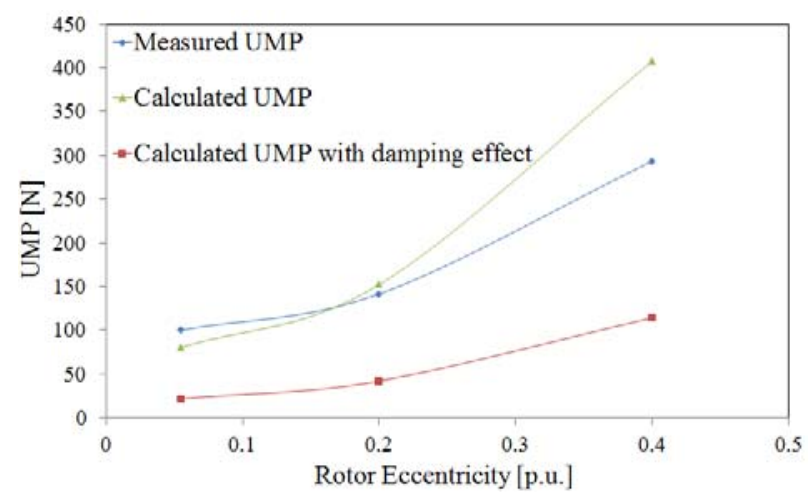

Fig. 12. Comparison of calculated and measured UMP with a variation of eccentricity at open circuit test, and comparison of calculated UMP with and without damping effect.

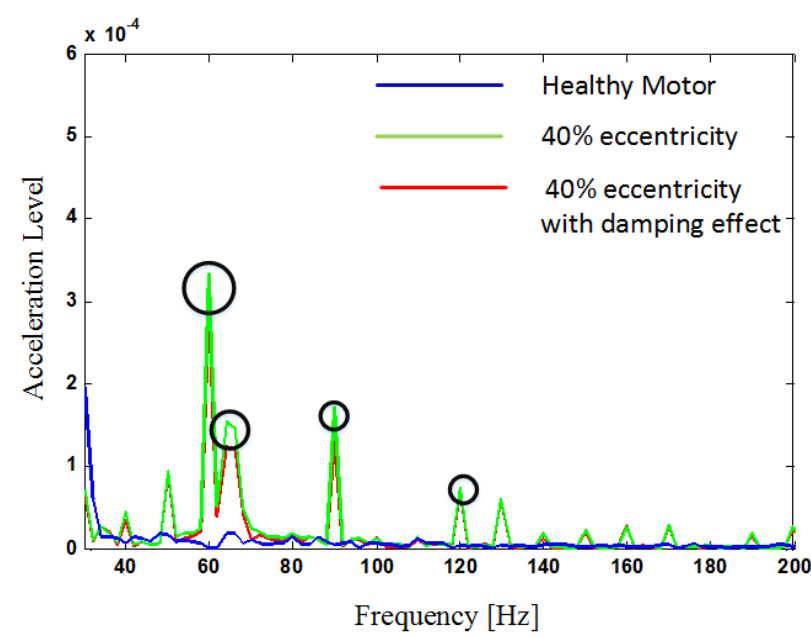

Fig. 13. Stator frame vibration zoom spectrum for a unifrom airgap, and $40 \%$ static eccentrcity.

\section{CONCLUSIONS}

This paper has developed the theory for calculating the EMFs induced into pole-specific search coils in a 4-pole wound rotor induction machine. A rig was constructed that can measure the search coil voltages and set the rotor in a non-centered manner. A 4-pole wound rotor induction motor was adapted to include pole-specific windings with 2 and 6 poles and it was illustrated that monitoring the voltage in these can be used to assess the rotor eccentricity. However, the running light (open circuit) and locked rotor tests show that the search coil voltages are very much a function of loading. In addition these windings can be used to inject current to adjust and damp the UMP. A method of incorporating damper windings in order to reduce the UMP and, hence, bearing wear has been assessed.

The practical applicability of the research is linked to large machines with low critical speeds which can be inaccessible, for example, the doubly-fed induction generator (DFIG) 
which is used in many wind turbine generators. Obviously, There is small level of static eccentricity existing even in newly manufactured machines due to the method of manufacturing and assembly. It can result in steady unbalanced one direction UMP. The paper also developed impedance matrices for both condition monitoring and UMP calculation and further work will be to program the control matrix and compare to experimental measurement when active UMP control is used.

\section{ACKNOWLEDGMENT}

The authors would like to sincerely thank Al Hussein Bin Talal University for financial support given through a doctoral scholarship to the student Ahmad Salah.

\section{REFERENCES}

[1] K. Mostafa, M. A. Mueller, and Q. Jiang, "Bearing wear detection and rotor eccentricity calculation in radial flux air-gap winding permanent magnet machines," IET PEMD Conference, 2014, pp. 1-6.

[2] M. Michon, K. Atillah, and G. Johnstone, "Effects of unbalanced magnetic pull in large permanent magnet machines," IEEE ECCE, 2014, pp. 4815-4820.

[3] B.M. Ebrahimi and J. Faiz, "Magnetic field and vibration monitoring in permanent magnet synchronous motors under eccentricity fault," IET Electr. Power Appl., vol. 6, no. 1, pp. 35-45, 2012.

[4] L. Wu, X. Huang, T. G. Habetler, and R. G. Harley, "Eliminating load oscillation effects for rotor eccentricity detection in closed-loop driveconnected induction motors," IEEE Trans. Power Elec., vol. 22, no. 4, pp. 15431551, 2007.

[5] D.-J. Kim, H.-J. Kim. J.-P. Hong, and C.-J. Park, "Estimation of acoustic noise and vibration," IEEE Trans. Magn., vol. 50, no. 2, Article\#: 7021204, 2014.

[6] D. G. Dorrell, "Sources and characteristics of unbalanced magnetic pull in 3-phase cage induction motors with axial-varying rotor eccentricity," IEEE Trans. Ind. Appl., vol. 47, no. 1, pp. 12-24, Jan./Feb. 2011.

[7] D. G. Dorrell, J. Shek, M.-F. Hsieh, and M. A. Mueller, "Damper windings in induction machines for reduction of unbalanced magnetic pull and bearing wear," IEEE Trans. Ind. Appl., vol. 49, no. 5, pp. 2206-2216, 2013.

[8] A. Chiba, T. Fukao, and M. Rahman, "Vibration suppression of a flexible shaft with a simplified bearingless induction motor drive," IEEE Trans. Ind. Appl., vol. 44, no. 3, pp. 745-752, 2008.

[9] A. Laiho, A. Sinervo, J. Orivuori, K. Tammi, A. Arkkio, and K. Zenger, "Attenuation of harmonic rotor vibration in a cage rotor induction machine by a self-bearing force actuator," IEEE Trans. Magn., vol. 45, no. 12 , pp. $1588-1598,2009$.

[10] A. Sinervo, A. Laiho, and A. Arkkio, "Low frequency oscillation in rotor vibrtion of a two-pole induction machine with extra four-pole stator winding," IEEE Trans. Magn., vol. 47, no. 9, pp. 22922302,2011 .

[11] A. Burakov and A. Arkkio, "Low-order parametric force model for eccentric rotor electrical machine with parallel connections in stator winding," IEE Proc. Power Appl., vol. 153, no. 4, pp. 592-600, 2006.

[12] D. G. Dorrell and O. Kayani, "Measurement and calculation of unbalanced magnetic pull in wound rotor induction machine," IEEE Trans. Magn., vol. 50, no. 11, Oct. 2014.

[13] S. Frushtenicht, E. Pittius, and H. O. Seinsch, "A diagnostic system for three-phase asynchronous machines," $4^{\text {th }}$ Int. Conf. on Electrical Machines and Drives, 13-15 Sept 1989, pp. 163-171.

[14] W. T. Thomson and A. Barbour, "On-line current monitoring and application of a finite element method to predict the level of static airgap eccentricity in three-phase induction motors," IEEE Trans. Energy Conv., vol. 13, no. 4, pp. 347-357, 1998.
[15] W. T. Thomson and D. G. Dorrell, "On-line current monitoring to diagnose airgap eccentricity in large three-phase induction motors industrial case histories verify the predictions", IEEE Trans. Energy Conv., vol. 14, no. 4, pp. 1372-1378, 1999.

[16] A. J. Ellison and S. J. Yang, "Effects of rotor eccentricity on acoustic noise from induction machines," Proc. of IEE, vol. 118, no. 1, pp. 174184, 1971.

[17] J. R. Cameron, W. T. Thomson, and A. B. Dow, "Vibration and current monitoring for detecting airgap eccentricity in large induction motors," Proc. of IEE, vol. 133, pt. B, no. 3, pp. 155-163, May 1986.

[18] R. Beguenane, M. Benbouzid, and H. El, "Induction motors thermal monitoring by means of rotor resistance identifications," IEEE Trans. Energy Conv., vol. 14, no. 3, pp. 566 -570, 1999.

[19] J. A. Penman, J. R. Smith, and W.E. Bryan, "Software based approach to machine condition monitoring system," $2^{\text {nd }}$ Int. Conf. on Electrical Machine Design and Applications, 1985, pp. 1-9.

[20] P. J. Tavner, L. Ran, J. Penman, and Howard Sedding, Condition Monitoring of Rotating electrical machines, IET publishers, UK, 2008.

[21] P. Vas, Parameter Estimation, Condition Monitoring and Diagnosis of Electrical machines, Clarendon Press, UK, 1993.

[22] D.-H. Hwang, S.-B. Han, B.-C. Woo, J.-H. Sun, D.-S. Kang, B.-K. Kim and Y.-H. Cho, "Detection of air-gap eccentricity and broken rotor bar conditions in a squirrel-cage induction motor using the radial flux sensor," Journal of Applied Physics, vol. 103, pp. 07F131-3, 2008.

[23] S. P. Verma and R. Natarajan, "Effects of eccentricity in induction motors," Int. Conf. on Electric Machines, 1982, pp. 930-933.

[24] D.G. Dorrell, W. T. Thomson, and S. Roach, "Analysis of airgap flux, current, and vibreation signals as a function of the combination of static and dynamic airqap eccentricity in 3 phase induction motors, "IEEE Trans. Ind. Appl., vol. 33, no. 1, pp. 24-25, Jan/Feb. 1997.

[25] W. T. Thomson, D. Rankin, and D. G. Dorrell, "On-line current monitoring to diagnose airgap eccentricity in large three-phase induction motors - industrial case histories verify the predictions," IEEE Trans. Energy Conv., vol. 14, no. 4, pp. 1372-1378, Dec. 1999.

[26] L. Frosini, A. Borin, L. Girometta, and G. Venchi., "Development of a leakage flux measurement system for condition monitoring of electrical drives, "IEEE Int. Symp. On Diagn. for Electric Machines, Power Electronics \& Drives (SDEMPED), 2011, pp. 356-363.

[27] A. Ceban, R. Pusca, and R. Romary, "Study of rotor faults in induction motors using external magnetic field analysis, "IEEE Trans. Ind. Electr., vol. 59, pp. 2082-2093, 2012.

[28] D.-H. Hwang, J.-H Chang, D.-S. Kang, J.-H. Lee, and K.-H. Choi., “A method for dynamic simulation and detection of air-gap eccentricity in induction motors by measuring flux density, "1 $12^{\text {th }}$ IEEE CEFC Conference, 2006, p. 69.

[29] D. G. Dorrell and A. Salah, "Detection of rotor eccentricity in wound rotor induction machines using pole-specific search coils," IEEE Trans. Magn., vol. 51, no. 11, article 8111604, Nov. 2015.

[30] D. G. Dorrell, Unbalanced Magnetic Pull in Cage Induction Machines, PhD Thesis, University of Cambridge, UK, 1993.

[31] A. C. Smith and D. G. Dorrell, "The calculation and measurement of unbalanced magnetic pull in cage induction motors with eccentric rotors. Part 1: Analytical model", Proc. IEE Electric Power Applications, vol. 143, no. 3, pp. 193-201, 1996.

[32] J. Fletcher and J. Yang, "Introduction to Doubly-Fed Induction Generator for Wind Power Applications," Paths to Sustainable Energy, Elsevier, 2010, DOI: 10.5772/12889. 